\title{
Künstliche Intelligenz - Fluch oder Segen?
}

Der technische Fortschritt, ein ständiger Begleiter unserer Gesellschaft, hat uns in den letzten Jahren gerade im Bereich der Informationstechnologie mit vielen Neuerungen konfrontiert. So war in den 80er Jahren der Desktop-PC das non-plus-ultra der EDV, ab Mitte der 90er Jahre wurde das Internet erschwinglich und einige Jahre später nahm dann die Entwicklung des Mobiltelefons an Fahrt auf - heute ist die Nutzung des Internets über Smartphone und Tablet überall und zu jeder Zeit ubiquitär, also normaler Zeitvertreib. Dabei haben die Mobiltelefone heute bereits mehr Rechenleistung, als damals ganze Rechenzentren.

Mit dem technischen Fortschritt wurde auch das Thema der künstlichen Intelligenz aufgeworfen. Der Begriff der künstlichen Intelligenz wurde als Schreckgespenst der sich emanzipierenden intelligenten Maschine zunächst durch Profanliteratur, wie Kampfstern Galactica, Terminator oder Matrix, allgemein bekannt, diente aber auch zunehmend als Werbeschlagwort für Software, denn jede drittklassige Softwarelösung versprach eine neuartige, herausragende künstliche Intelligenz, sei es das neue Strategiespiel, der Vokabeltrainer für den Nachwuchs oder die Bundesligaergebnistabelle für Papas Homecomputer. Bei genauer Betrachtungsweise entpuppte sich dann aber diese gepriesene Kl als lahme Chimäre, als ein Potemkinsches Dorf.

Mittlerweile ist KI keine „Luftnummer" mehr. Die KI, also der selbstlernende Prozess, birgt die Möglichkeit, dass die Software in der Lage sein wird, nicht nur zu "Lernen", sondern auch eigene Lösungsansätze oder gar Ideen zu entwickeln. Der Ausblick ist: Automaten, die Probleme selbständig lösen, die sich selbst weiterentwickeln usw. Doch bei der Vorfreude auf ungeahnten Fortschritt ziehen am Firmament "dunkle Wolken" auf: Welche Gefahren bringt der Fortschritt? Was passiert, wenn die $\mathrm{Kl}$ sich nicht mehr so verhält, wie wir es von ihr erwarten. Diese Fragestellung ist jedoch nicht erst durch besagte Popularfilme bekannt. Bereits die Golem-Legende des Hochmittelalters nahm sich dieses Topos an: Der Golem, ein Kunstwesen aus Lehm, der der jüdischen Gemeinde Prags helfen sollte, die erfundenen Vorwürfe des Kindesmords zu widerlegen, entzog sich später der Kontrolle seines Herrn. Das sind aber Zukunftsszenarien. Unmittelbar drohen andere Gefahren, die nicht zu unterschätzen sind: Ein auf künstlicher Intelligenz beruhender Prozess kann eine umfassende Datenverarbeitung und -auswertung ermöglichen. Der Alp der Vorratsdatenspeicherung wird durch eine funktionierende Kl erst Realität. Wie wir außerdem aus der heutigen Bewertung der Arbeit des Ministeriums für Staatssicherheit der DDR wissen, hat diese „Behörde" seinerzeit einen gigantischen "Schatz" an Daten angehäuft, diesen aber aufgrund fehlender funktionierender Auswertung kaum nutzen können. Aber genau die Auswertung gigantischer Datensätze ist mittels funktionierender $\mathrm{KI}$ nunmehr möglich, weil Informationen aus den diversen Quellen abgeglichen werden können. Was passiert aber, wenn Datensätze aus Social-Media, wie facebook, tumblr u.ö, mit Bewegungsdaten aus GPS-tracking und Paycards, Einkaufsverläufe aus Amazon, verbunden werden und zu einer Gesamtdatenbank zusammengeführt werden?

An diesem Negativbeispiel wird deutlich, dass hier nicht die Technik die Gefahr birgt, sondern der Einsatz der Technik durch den Menschen. Denn diese Gesamtdatenbanken (big data) entstehen nicht automatisch, sondern weil der Anwender genau dies bezweckt, denn mit diesen Daten lässt sich erheblicher Gewinn erzielen. Umso wichtiger ist, dass der Datenschutz sich dieser Gefahr bewusst ist und der Gesetzgeber uns die Mittel an die Hand gibt, den Gefahren für die Betroffenen zu begegnen, damit Datenschutz das bleibt, was er ist, nämlich das Recht auf informationelle Selbstbestimmung.

\section{Dominik Bleckmann}

